\title{
Canvassing the External Applications of Snuhi : A Classical Memoir
}

\author{
Review Article
}

\section{Shashi Gupta ${ }^{*}$, Rabinarayan Acharya ${ }^{2}$}

1. PG Scholar, 2. Professor \& Head,

Department of Dravyaguna, IPGT \& RA, Gujarat Ayurved University, Jamnagar -361008.

\begin{abstract}
Classical texts of Ayurveda, basing upon the presence of spines, describes two types of Snuhi i.e, one having excess of spines and the other having less number of spines. The plant having more number of spines are considered as Shrestha (superior). Various parts of Snuhi were administered either externally or internally in multi dosage forms to combat varied disease conditions. Euphorbia neriifolia has been considered as the botanical equivalent of snuhi. A single hand data is unavailable on the usage of Snuhi, externally in various anomalies. This review portrays the wide usage of Snuhi in the form of external administration from 52 different classical texts consisting of treatise and compendia. It is observed that about 253 formulations, in 20 dosage forms, have been recommended, almost 62 varied diseases. Among these disease conditions, Kustha (Skin disorders) is maximum, followed by Arsha (Haemorhoids) and Vatavyadhi (Neuro-muscular anomalies) etc. where latex part of Snuhi has been indicated in external application.
\end{abstract}

Keywords: Euphorbia neriifolia, External applications, Skin diseases, Snuhi, Upavisha.

\section{Introduction:}

Drugs of herbal origin dominate among all other group of drugs being recommended by Ayurvedic Pharmacopoeia. Among the drugs of herbal origin many drugs, due to their specific actions, are categorized as Visha (Poisonous), Upavisha (Semipoisnous group of drugs). Snuhi has been categorized under Upavisha (Semi-poisnous) category (1). Snuhi, based on presence of spines, described two types, one having excess of spines and the other having less number of spines. The plant having more number of spines are considered as Shrestha (Superior) (2). Snuhi has been attributed with numerous pharmaceutical and therapeutic claims in various treatise and compendia. Inspite of its inclusion in semipoisonous group, it has been advocated with external and internal applications in various formulations, for different ailments.

Basically two species, Euphorbia neriifolia Linn. and Euphorbia antiquorum Linn. are taken to be the botanical sources of two varieties of Snuhi i.e. Thuhara and Tridhara thuhara. Dravyaguna scholars, in recent texts, categorized Snuhi under two varieties i.e. Kanda Snuhi (Euphorbia tirucalli Linn.) and Patra Snuhi (Euphorbia neriifolia Linn.). (64) Pharmacological properties and actions of drugs used in Ayurveda has

\section{*Corresponding Author:}

\section{Shashi Gupta}

PG scholar Department of Dravyaguna,

IPGT \& RA, Gujarat Ayurved University,

Jamnagar -361008.

E-mail ID: shashi.gpt@gmail.com been described in various classical treatise and compendia and texts related to Rasashastra. These Pharmacopoeias predominantly deals with drugs of herbal and herbo-mineral origin respectively. Further, there are certain texts which also contain formulation of both herbs and herbo-mineral and being recommended in various disease condition known in Chikitsa grantha (Compendia). Single handed information on the external application of usage of Snuhi, from all the above mentioned texts, is not available. Hence, this review portrays the wide usage of Snuhi in, the form of external administrations.

\section{Materials and methods:}

In this review, information from various texts like Charaka Samhita (Ch.S.) (3); Sushruta Samhita (Su.S.) (4); Ashtanga Samgraha (A.S.) (5); Ashtanga Hridaya (A.H.) 6); Kashyapa Samhita (Ka.S.) (7); Bhela Samhita (Bh.S.) (8); Harita Samhita (Ha.S.) (9); Vrindamadhava (Vr.Md.) (10); Chakradutta (C.D.) (11); Gadanigraha (G.Ni.) (12); Vangasena (V.S.) (13); Sharangdhara Samhita (Sh.S.) (14); Bhavaprakasha Samhita (Bh.P.S.) (15); Yogaratnakara (Y.R.) (16); Bruhata Nighantu Ratnakara (Bh.Ni.Rt.) (17); Bhaishyajya Ratnavali (Bh.Rt.) (18); Sahasra Yoga (S.Y.) (19); Bharata Bhaisajya Ratnakara (B.B.R.) (20); Chikitsa Kalika (Ch.Ka.) (21); Chamatkara Chintamani (Ch.Chi.) (22); Siddhabheshaja Manimala (Si.B.M.) (23); Vaidya Jivana (V.J.) (24); Vaidya Manorama (Vd.M.) (25) Rasendra Mangala (R.Mn.) (26); Rasa Hridaya Tantra (R.H.T.) (27); Rajamartanda (R.M.) (28); Rasarnava Nama Rasatantrama (Rr.Rt.) (29); Rasendra 
Chudamanai (Rs.Chu.) (30); Rasa Prakasha Sudhakara (Rs.Pr.) (31); Rasa Ratna Samucchaya (R.R.S.) (32); Rasa Paddhati (R.P.) (33); Ananda Kanda (A.K.) (34); Rasa Chintamani (Rs.Ch.) (35); Rasamanjiri (R.M.) (36); Rasendra Chintamani (Rs.Chn) (37); Rasendra Sara Samgraha (R.S.S.) (38); Rasa Kamadhenu (R.K.) (39); Yogatarangini (Y.T.) (40); Arka Prakasha (Ar.Pr.) (41); Ayurveda Prakasha (Ay.Pr.) (42); Brihata Rasaraja Sundara (B.R.S.) (43); Rasaraja Mahodadhi (R.R.M.) (44); Rasa Jala Nidhi (R.J.N.) (45); Rasayoga Sagara (R.Y.S.) (46); Rasamitra (R.Mi.) (47); Rasopnishata (R.N.) (48); Rasatarangini (R.T.) (49); Rasendra Sambhava (Rs.Sm.) (50); Abhinava Navjeevan (Ab.Nv.) (51); Rasa Kaumidi (Rs.Ku.) (52); Rasa Sanketa Kalika (Ra. S. K.) (53); Rasa Dipika (R.D.) (54) has been compiled and noted down.

Snuhi as a single drug or as content in formulations in the above mentioned 52 classical texts were critically analysed with repect to formulations, variety of Snuhi, parts used, their dosage form and indications. Due to the vast data, adhikara - wise formulations were categorised and accordingly tables have been prepared to present the information. (Table 1 -14).

The Sanskrit terms of formulations have been expressed into convenient English language using accepted dictionaries (55). English equivalent of various disease conditions were referred from AFI (Ayurvedic Formulary of India) (56).

Various abbbreviations were used for various dosage forms such as Lepa (Lp), Taila (Tl) A bhyanga (Ab), Varti (Vr), Udvartana (Uv), Nasya (Ns), Bandhana (Bn), Kshira (Ks), Swedana (Sd), Sinchana (Sn), Dharana, Ghrita (Gr), Anjana, Kalka (Kl), Dhoopa (Dp), Purana (Pr), Basti (Bs), Churna (Cr), Snana and Sparsha.

\section{RESULTS:}

On keen observation, it is noted that Snuhi has been used as an ingredient in a total of 253 formulations. Different parts of the plant Snuhi are used in various dosage forms in many disease conditions. The details of parts used, dosage forms and indications, are as below.

\section{Parts:}

Almost all parts of Snuhi (i.e latex, leaves, stem, fruit, bark, whole plant, etc.) have been included as ingredients in these formulations. As external application among them Kshira (latex) accounts for maximum (216), followed by Patra (leaf) in 16, kanda (stem) in 13 and moola (root) in 10 formulations and one each in phala (fruit) and panchanga (whole plant) ( Table1-14)

\section{Dosage forms:}

Maximum of formulations, in external applications, include Lepa (Pasting) (Lp) (111) and Taila (oil) (Tl) (48) followed by Abhyanga (Oleation) (Ab) (34), Varti (Anal suppository) (Vr) (21), Udvartana (Herbal massage powder therapy) (Uv) (9), Nasya (Snuffing) (Ns) (7), Bandhana (Bandage) (Bn) (6), Kshira (Latex) (Ks) (5), Swedana (Sudation) (Sd)
(5), Sinchana (Sprinkling media) (Sn) (3), Dharana (3), Ghrita (Medicated ghee) (Gr) (2), Anjana (Collyrium) (2), Kalka (Paste) (Kl) (2), Dhoopa (Fumigation) (Dp) (2), one each of Purana (Ear drop) (Pr), Basti (Enema therapy) (Bs), Churna (Powder) (Cr), Snana (Bath) and Sparsha (Touch).

Indications:

Kustha (Skin disorders) - Ayurveda considers various skin disorders under a single disease condition namely Kushtha. Snuhi has been indicated in Kustha (48), among the parts used, Snuhi kshira (36) has been used maximum in skin disorders in dosage form of oil (14) and lepa (16). (Table 1)

Arsha (haemorrhoids) - Total 28 formulations, Kshira (latex) as parts use, has been reported maximum, among the dosage forms, lepa is maximum used in management of Arsha. (Table 2)

Vatavyadhi (Neuro-muscular anomalies) - Different parts of Snuhi are used in 13 formulations, Kshira (latex) has been reported maximum. Snuhi has been used mainly in external oleation and fomentation therapies. (Table 3)

Shwitra (Leucoderma / Vitiligo) - Total 12 formulations, Snuhi as an ingredient, reported for the management of Shwitra where, Kshira (latex) has been reported maximum, and in dosage forms, lepa is maximum.(Table 4)

Bhagandara (Fistula-in-ano) - It is one of the anorectal disorders, having fistular tract, with severe pain. To manage this disease ten formulations, with Kshira (latex) of Snuhi as a major ingredient, in oil and varti dosage form has been recommended in classics texts. (Table 5)

Dadru (Ring worm or Fungal infection) - To manage this disease 10 formulations, having Snuhi as an ingredient, Kshira (latex) maximum parts use and lepa as maximum dosage form has been reported. (Table 6)

Vrana (Ulcer) - Total 8 formulations are having Snuhi as an ingredient, Kshira (latex) maximum parts use and in dosage forms, lepa as maximum dosage form has been reported. (Table 7)

Nadi vrana (Sinus) - Seven formulations are having Snuhi as an ingredient, Kshira (latex) maximum parts use and, varti as dosage forms in management of Nadivrana. (Table 8)

Visha (Poison) - Total 6 formulations are having Snuhi as an ingredient. Among the parts used, Kshira (latex) has been reported maximum and in dosage forms, lepa is maximum used in the management of Visha. (Table 9)

Granthi (Cyst) - Total 6 formulations are having Snuhi as an ingredient. Among the parts used, Kshira (latex) has been reported maximum and in dosage forms, lepa 
is used maximum in the management of Granthi. (Table $10)$.

Galganda (Goitre) - Total 6 formulations are having Snuhi as an ingredient. Among the parts used, Kshira (latex) has been reported maximum and in dosage forms, lepa is maximum used in the management of Galganda. (Table 11)

Pama (Eczema) - Total 6 formulations are having Snuhi as ingredient. Among the parts used, Kshira (latex) has been reported maximum and in dosage forms, udvartana is used maximum in the management of Pama. (Table 12)

Other indications include four formulations each in Pada roga (Diseases of sole), Raktagulma (Uterine tumour), Stri roga (Disease of female genital organs) and Vicharchika (Dry and weeping eczema); Three formulations contains Snuhi Apachi (Chronic lymphadenitis), Arushika (Seborrhea), Gandamala (Srofula), Karna roga (Ear disease), Kitibha (Psoriasis), Masaka (Elevated mole), Sotha (Oedema), Udara (Ascites), Upadansa (Gonorrhoea) and Vatarakta (Gout);
Two formulations contains Snuhi Arbuda (tumour), Galsundi (Uvulitis), Garbhasalya, Indralupta (Baldness), Kachu (Scabies), Khalitya (Alopecia), Kshara karma, Sarva roga, Shiroroga (Headache), Snayuka (Dracontiasis), Udavarta (Abdominal disease characterized by retention of faeces), Visarpa (Erysipelas) and rest one formulations contains Snuhi each in Agnidaha (Burning sensation), Antra vidradhi (Internal abcess), Apara patana, Apasmara (epilepsy), Bhagna (Bone fracture), Garbhini (Pregnancy), Gulma (Intestinal lump), Kandu (Itching), Kantaka vidha, Karnika patana, Kesa ranjana, Khalli (Cramps of ankle), Krimidanta (Dental caries), Kunakha (Onychia), Masurika (Small pox), Mrita garbha, Mudha garbha (Foetal malpresentation), Prasuta, Romshatana, Shlipada (Filariasis), Unmada (Psychosis), Urdhwajatru gata kapha roga, Urustambha (Spasticity of thigh), Visphota (Eruptions) and Visuchika (Cholera). Total 56 formulations have Snuhi as ingredient in different panchakarma. Among the parts used, Kshira (latex) has been reported maximum and in dosage forms, Abhyanga is maximum. (Table13,14).

Table 1: Snuhi as an ingredient of various formulations indicated in Kustha (Skin disorders)

\begin{tabular}{|l|l|l|l|l|l|}
\hline Sr. No. & Formulation & Dosage Forms & Rogadhikara & Part used & References \\
\hline 1 & Mahavajraka taila & $\mathrm{Ab}$ & Kustha & Kshira & $4,5,6$ \\
\hline 2 & Kustha kalanala taila & $\mathrm{Ab}$ & Kustha & Kshira & 20 \\
\hline 3 & Kusthadi taila & $\mathrm{Ab}$ & Kustha & Kshira & $6,17,20$ \\
\hline 4 & Marichyadi taila & $\mathrm{Ab}$ & Kustha & Kshira & $11,13,20$ \\
\hline 5 & Kusthahara yoga & $\mathrm{Ab}$ & Kustha & Kshira & 44 \\
\hline 6 & Vajri taila & $\mathrm{Ab}$ & Kustha & Kshira & 20,32 \\
\hline 7 & Bhanu taila & $\mathrm{Ab}$ & Kustha & Kshira & 20,39 \\
\hline 8 & Vajraka taila & $\mathrm{Ab}$ & Kustha & Kshira & $4,5,10,13,14,16,17,18,20$ \\
\hline 9 & Mahamarichadi taila & $\mathrm{Ab}, \mathrm{Ns}$ & Kustha & Kshira & $10,11,12,18,39$ \\
\hline 10 & Mahamarichadi taila & $\mathrm{Ab}, \mathrm{Ns}$ & Kustha & Kshira & 15 \\
\hline 11 & Masyadi lepa & $\mathrm{Lp}$ & Kustha & Kshala (Bark) & 3 \\
\hline 12 & Sajiksharadi lepa & $\mathrm{Lp}$ & Kustha & Kshira & 4 \\
\hline 13 & Tuthadi lepa & $\mathrm{Lp}$ & Kustha & Kshira & 4 \\
\hline 14 & Kustha kalanala taila & $\mathrm{Lp}$ & Kustha & Kshira & 12 \\
\hline 15 & Chakrangabeejadi yoga & $\mathrm{Lp}$ & Kustha & Kshira & 10,11 \\
\hline 16 & Gunjadi lepa & $\mathrm{Lp}$ & Kustha & Kshira & 20 \\
\hline 17 & Grihadhoomadi lepa & $\mathrm{Lp}$ & Kustha & Kshira & 12,20 \\
\hline 18 & Vishadi lepa & $\mathrm{Lp}$ & Kustha & Kshira & 18,20 \\
\hline 19 & Katuka alabuadi lepa & $\mathrm{Lp}$ & Kustha & Moola & 20 \\
\hline 20 & Bhallatakadi taila & $\mathrm{Lp}$ & Kustha & Moola, Kshira & 6,12 \\
\hline 21 & Bhallatakadi lepa & $\mathrm{Lp}$ & Moola, Kshira & $5,10,11,18,50$ \\
\hline 22 & Snuka lepa & $\mathrm{Lp}$ & Patra & 20 \\
\hline
\end{tabular}


International Journal of Ayurvedic Medicine, 2017, 8(2), 45-57

\begin{tabular}{|c|c|c|c|c|c|}
\hline Sr. No. & Formulation & Dosage Forms & Rogadhikara & Part used & References \\
\hline 23 & Putikadi lepa & Lp & Kustha & Patra & $4,10,12,20$ \\
\hline 24 & Shwitragajasigha taila & $\mathrm{Lp}, \mathrm{Ab}$ & Kustha & Kshira & 35 \\
\hline 25 & Vishadi yoga & $\mathrm{Sd}$ & Kustha & Khsira & 10,12 \\
\hline 26 & Mahamartanda taila & $\mathrm{Tl}$ & Kustha & Kanda & 22,34 \\
\hline 27 & Gunja mooladi taila & $\mathrm{Tl}$ & Kustha & Kshira & 12 \\
\hline 28 & Visha taila & $\mathrm{Tl}$ & Kustha & Kshira & 12 \\
\hline 29 & Sada bindu taila & $\mathrm{Tl}$ & Kustha & Kshira & 18 \\
\hline 30 & Kacchuradi taila & $\mathrm{Tl}$ & Kustha & Kshira & 19 \\
\hline 31 & Chitraka taila & $\mathrm{Tl}$ & Kustha & Kshira & 20,39 \\
\hline 32 & Snuhiadi taila & $\mathrm{Tl}$ & Kustha & Kshira & $13,20,32$ \\
\hline 33 & Kusthahara taila & $\mathrm{Tl}$ & Kustha & Kshira & 32,35 \\
\hline 34 & Brihatamarichadi taila & $\mathrm{Tl}$ & Kustha & Kshira & $10,11,18,39$ \\
\hline 35 & Mahatrinaka taila & $\mathrm{Tl}$ & Kustha & Patra & 20 \\
\hline 36 & Kandarpa sara taila & $\mathrm{Tl}$ & Kustha & Patra & 18,20 \\
\hline 37 & Prapunnadi udvartana & Uv & Kustha & Kshira & 11 \\
\hline 38 & Gunjadi udvartana & Uv & Kustha & Kshira & 16 \\
\hline 39 & Snuhyadi udvartana & Uv & Kustha & Kshira & 25 \\
\hline 40 & Vajra shekhara rasa & Uv & Kustha & Kshira & 46 \\
\hline 41 & Nishadi yoga & Uv & Kustha & Patra & 10 \\
\hline 42 & Gandirakadi taila & $\mathrm{Tl}$ & Mandala kustha & Kshira & 11 \\
\hline 43 & Rasatankadi lepa & Lp & Charma kustha & Kshira & 39 \\
\hline 44 & Gandhaka taila & $\mathrm{Tl}$ & Kshudra kustha & Kshira & 49 \\
\hline 45 & Erandabeejadi lepa & Lp & Sidhma & Kshira & 51 \\
\hline 46 & Mahatrina taila & $\mathrm{Tl}$ & Twaka roga & Patra & 11,18 \\
\hline
\end{tabular}

Table 2: Snuhi as an ingredient of various formulations indicated in Arsha (Haemorrhoids)

\begin{tabular}{|l|l|l|l|l|l|}
\hline Sr. No. & Formulation & Dosage Forms & Rogadhikara & Part used & References \\
\hline 1 & Brihata kasisadi taila & $\mathrm{Ab}$ & Arsha & Kshira & $15,20,51$ \\
\hline 2 & Kasisadi taila & $\mathrm{Ab}$ & Arsha & Kshira & $5,12,18,20,35,39$ \\
\hline 3 & Ksharasootra & $\mathrm{Bn}$ & Arsha & Kshira & $10,11,13,15,18,20,39$ \\
\hline 4 & Rajni churna yoga & $\mathrm{Bn}$ & Arsha & Kshira & 17 \\
\hline 5 & Snukapatra yoga & $\mathrm{Bn}$ & Arsha & Patra & 20 \\
\hline 6 & Arkapaya adi lepa & Lp & Arsha & Kanda & 6 \\
\hline 7 & Gauri pashana lepa & Lp & Arsha & Kanda & 20 \\
\hline 8 & $\begin{array}{l}\text { Haridra churnadi } \\
\text { pralepa }\end{array}$ & Lp & Arsha & Kshira & $3,15,16,17,20,39$ \\
\hline 9 & Shirisa-beejadi pralepa & Lp & Arsha & Kshira & $3,8,12,35,40,50$ \\
\hline 10 & Arka-kshiradi pralepa & Lp & Arsha & Kshira & $3,6,11,12,16,17,18,32$ \\
\hline 11 & Snuhi kskiradi lepa & Lp & Arsha & Kshira & $4,5,6,11,17,39,49$ \\
\hline
\end{tabular}




\begin{tabular}{|l|l|l|l|l|l|}
\hline Sr. No. & Formulation & Dosage Forms & Rogadhikara & Part used & References \\
\hline 12 & Sudhadi lepa & $\mathrm{Lp}$ & Arsha & Kshira & $20,39,49$ \\
\hline 13 & Kulisadi lepa & $\mathrm{Lp}$ & Arsha & Kshira & 6,28 \\
\hline 14 & Snuka kshira pistadi lepa & $\mathrm{Lp}$ & Arsha & Kshira & 6 \\
\hline 15 & Kuleeradi lepa & $\mathrm{Lp}$ & Arsha & Kshira & 6 \\
\hline 16 & Sourmikadi lepa & $\mathrm{Lp}$ & Arsha & Kshira & 8 \\
\hline 17 & Swarnakshiradi lepa & $\mathrm{Lp}$ & Arsha & Kshira & 8 \\
\hline 18 & Katu tumbyadi lepa & $\mathrm{Lp}$ & Arsha & Kshira & 17 \\
\hline 19 & Arshohara lepa & $\mathrm{Lp}$ & Arsha & Kshira & 20 \\
\hline 20 & Swarchikado lepa & $\mathrm{Lp}$ & Arsha & Kshira & 20 \\
\hline 21 & Snuhikshira lepa & $\mathrm{Lp}$ & Arsha & Kshira & 20 \\
\hline 22 & Chitrakadi taila & $\mathrm{Lp}$ & Arsha & Kshira & 12,20 \\
\hline 23 & Pippalyadi lepa & $\mathrm{Lp}$ & Arsha & Kshira & $15,18,20$ \\
\hline 24 & Gaja asthiadi taila & $\mathrm{Lp} /$ Ab & Arsha & Kanda & 5 \\
\hline 25 & Mahanimbadi taila & $\mathrm{Tl}$ & Arsha & Kshira & 8 \\
\hline 26 & Putikaranjadi varti & $\mathrm{Vr}$ & Arsha & Kshira & 5 \\
\hline 27 & Jalinibeejadi varti & $\mathrm{Vr}$ & Arsha & Kshira & 12 \\
\hline 28 & Katutumbiadi varti & $\mathrm{Vr}$ & Arsha & Kshira & 12,20 \\
\hline
\end{tabular}

Table 3: Snuhi as an ingredient of various formulations indicated in Vatavyadhi

\begin{tabular}{|c|c|c|c|c|c|}
\hline Sr. No. & Formulation & Dosage Forms & Rogadhikara & Part used & References \\
\hline 1 & Vishatinduka taila & $\mathrm{Ab}$ & Vatavyadhi & Kshira & 18 \\
\hline 2 & Visha tindukataila & $\mathrm{Ab}$ & Vatavyadhi & Kshira & 20 \\
\hline 3 & Atharanga taila & $\mathrm{Ab}$ & Vatavyadhi & Patra & 44 \\
\hline 4 & Maha Narayana taila & $\mathrm{Ab}, \mathrm{Aj}, \mathrm{Ns}, \mathrm{Bs}$ & Vatavyadhi & Moola & 15 \\
\hline 5 & Snuhyadi lepa & Lp & Vatavyadhi & Kshira & 25 \\
\hline 6 & Mahavisha garbha taila & Lp & Vatavyadhi & Kshira & 16,17 \\
\hline 7 & Erandadi yoga & $\mathrm{Sd}$ & Vatavyadhi & Kshira & 16,17 \\
\hline 8 & Prasarini taila & $\mathrm{Tl}$ & Vatavyadhi & Kshira & 17 \\
\hline 9 & Chinchadi taila & $\mathrm{Tl}$ & Vatavyadhi & Kshira & 19 \\
\hline 10 & $\begin{array}{l}\text { Maha Lakshmi Narayana } \\
\text { taila }\end{array}$ & $\mathrm{Tl}$ & Vatavyadhi & Kshira & 16,17 \\
\hline 11 & Visha garbha taila & $\mathrm{Tl}$ & Vatavyadhi & Kshira & 17,20 \\
\hline 12 & Lakuchadi siddha taila & Lp & Avabahuka & Kshira & 25 \\
\hline 13 & Lakwahara taila & $\mathrm{Ab}$ & Lakwa & Kshira & 44 \\
\hline
\end{tabular}

Table 4: Snuhi as an ingredient of various formulations indicated in Shwitra(Vertiligo)

\begin{tabular}{|l|l|l|l|l|l|}
\hline Sr. No. & Formulation & Dosage Forms & Rogadhikara & Part used & References \\
\hline 1. & Vishadi lepa & Lp & Shwitra & Kshira & 11 \\
\hline 2. & Gomutradi lepa & Lp & Shwitra & Kshira & 5,11 \\
\hline
\end{tabular}




\begin{tabular}{|l|l|l|l|l|l|}
\hline Sr. No. & Formulation & Dosage Forms & Rogadhikara & Part used & References \\
\hline 3. & Bhadra udambaradi lepa & Lp & Shwitra & Moola & 12 \\
\hline 4. & Katukadi taila & Lp & Shwitra & Moola,kshira & 13 \\
\hline 5. & Putikadi lepa & Lp & Shwitra & Patra & $5,6,11,18$ \\
\hline 6. & Kunatadi lepa & Lp & Shwitra & Patra & 13 \\
\hline 7. & Puner lepa & Lp & Shwitra & Patra & 39 \\
\hline 8. & Mutrika rasakriya & Lp & Shwitra & Phala & 5 \\
\hline 9. & Mahavajraka taila & $\mathrm{Tl}$ & Shwitra & Kshira & 6 \\
\hline 10. & Aruskaradi taila & $\mathrm{Tl}$ & Shwitra & Kshira & 21 \\
\hline 11. & Shwitra hara taila & $\mathrm{Tl}$ & Shwitra & Kshira & 20,35 \\
\hline 12. & Vajraka taila & $\mathrm{Tl}$ & Shwitra & Kshira & 20 \\
\hline
\end{tabular}

Table 5: Snuhi as an ingredient of various formulations indicated in Bhagandara (Fistula)

\begin{tabular}{|l|l|l|l|l|l|}
\hline Sr. No. & Formulation & Dosage Forms & Rogadhikara & Part used & References \\
\hline 1. & Jyotismati adi taila & $\mathrm{Ab}$ & Bhagandara & Kshira & 6 \\
\hline 2. & Chitrakadi taila & $\mathrm{Ab}$ & Bhagandara & Kshira & 12,20 \\
\hline 3. & Ksharasootra & $\mathrm{Bn}$ & Bhagandara & Kshira & 49 \\
\hline 4. & Syandana taila & $\mathrm{Tl}$ & Bhagandara & Kanda & 20 \\
\hline 5. & Pippalyadi taila & $\mathrm{Tl}$ & Bhagandara & Kshira & 4 \\
\hline 6. & Trivritadi taila & $\mathrm{Tl}$ & Bhagandara & Kshira & 4 \\
\hline 7. & Vishyandana taila & $\mathrm{Tl}$ & Bhagandara & Kshira & $4,11,12,13$, \\
& & & & & $15,16,17,18,20$ \\
\hline 8. & Snuhi arkadugdhadi varti & $\mathrm{Vr}$ & Bhagandara & Kshira & 40 \\
\hline 9. & Snuhiadi varti & $\mathrm{Vr}$ & Bhagandara & Kshira & $11,12,13,18$ \\
\hline 10. & Kshara vartika & $\mathrm{Vr}$ & Bhagandara & Kshira & 49 \\
\hline
\end{tabular}

Table 6: Snuhi as an ingredient of various formulations indicated in Dadru

\begin{tabular}{|l|l|l|l|l|l|}
\hline Sr. No. & Formulation & Dosage Forms & Rogadhikara & Part used & References \\
\hline 1. & Aswaghnamooladi taila & Ab & Dadru & Kshira & 9 \\
\hline 2. & Dhatriadi lepa & Lp & Dadru & Kshira & 13 \\
\hline 3. & Chakramardadi lepa & Lp & Dadru & Kshira & 20 \\
\hline 4. & Darvyadi suryapaka taila & Lp & Dadru & Kshira & 12,20 \\
\hline 5. & Paradadi lepa & Lp & Dadru & Kshira & 18,39 \\
\hline 6. & Prapunatadi lepa & Lp & Dadru & Kshira & $13,16,17,20,51$ \\
\hline 7. & Gandilaki lepa & Lp & Dadru & Patra & 15 \\
\hline 8. & Gandiradi taila & Tl & Dadru & Kshira & 13 \\
\hline 9. & Dravyantadi taila & Tl & Dadru & Kshira & 20 \\
\hline 10. & Gunjadi yoga & Uv & Dadru & Kshira & 17 \\
\hline
\end{tabular}

Table 7: Snuhi as an ingredient of various formulations indicated in Vrana (Ulcer)

\begin{tabular}{|l|l|l|l|l|l|}
\hline Sr. No. & Formulation & Dosage Forms & Rogadhikara & Part used & References \\
\hline 1. & Aswaghnamooladi taila & $\mathrm{Ab}$ & Dadru & Kshira & 9 \\
\hline 2. & Dhatriadi lepa & Lp & Dadru & Kshira & 13 \\
\hline 3. & Umadi lepa & Lp & Pakva vrana & Kshira & 3 \\
\hline
\end{tabular}


Shashi Gupta et.al., Canvassing the External Applications of Snuhi: A Classical Memoir

\begin{tabular}{|l|l|l|l|l|l|}
\hline Sr. No. & Formulation & Dosage Forms & Rogadhikara & Part used & References \\
\hline 4. & Snuka patra yoga & Lp & Vrana & Patra & 20 \\
\hline 5. & Dasamooladi lepa & Lp & Vrana sotha & Kshira & 15 \\
\hline 6. & Dantyadi lepa & Lp & Vrana sotha & Kshira & 14,16 \\
\hline 7. & Jatipuspadi lepa & Lp & Vrana sotha & Kshira & 13,20 \\
\hline 8. & Arkadi lepa & Lp & Vrana sotha & Kshira & 44 \\
\hline
\end{tabular}

Table 8: Snuhi as an ingredient of various formulations indicated in Nadivrana (sinus)

\begin{tabular}{|l|l|l|l|l|l|}
\hline Sr. No. & Formulation & $\begin{array}{l}\text { Dosage } \\
\text { Forms }\end{array}$ & Rogadhikara & $\begin{array}{l}\text { Part } \\
\text { used }\end{array}$ & References \\
\hline 1. & Snuhiadi varti & $\mathrm{Vr}$ & Nadi vrana & Kshira & 13 \\
\hline 2. & Snuka kshiradi varti & $\mathrm{Vr}$ & Nadi vrana & Kshira & 20 \\
\hline 3. & Kumbhikadi taila & $\mathrm{Vr}$ & Nadi vrana & Kshira & 15 \\
\hline 4. & Badari phalatwakadi varti & $\mathrm{Vr}$ & Nadi vrana & Kshira & 20 \\
\hline 5. & Nadivranahara varti & $\mathrm{Vr}$ & Nadi vrana & Kshira & 44 \\
\hline 6. & Ghontaphaltwakadi Varti & $\mathrm{Vr}$ & Nadi vrana & Kshira & $4,6,11,12,40$ \\
\hline 7. & Jatyadi varti & $\mathrm{Vr}$ & Nadi vrana & Kshira & $11,12,13,16,20,40$ \\
\hline
\end{tabular}

Table 9: Snuhi as an ingredient of various formulations indicated in Visha

\begin{tabular}{|l|l|l|l|l|l|}
\hline Sr. No. & Formulation & Dosage Forms & Rogadhikara & Part used & References \\
\hline 1. & Sirisabeejadi lepa & Lp & Kukkura visha & Kshira & 18,50 \\
\hline 2. & Shrisa-phaladi lepa & Lp & Manduka visa & Kshira & $3,5,11,16,17,18$ \\
\hline 3. & Sirisabeejadi lepa & Lp & Manduka visha & Kshira & $3,11,16,17,18,40,50$ \\
\hline 4. & Sirisapuspadi yoga & Ns & Manduka visha & Kshira & 28 \\
\hline 5. & Ksharagada & Lp & Visha & Kshira & 20 \\
\hline 6. & Ghritadi lepa & Lp & Vrischika visha & Kshira & 50 \\
\hline
\end{tabular}

Table 10: Snuhi as an ingredient of various formulations indicated in Granthi (Cyst)

\begin{tabular}{|l|l|l|l|l|l|}
\hline Sr. No. & Formulation & Dosage Forms & Rogadhikara & Part used & References \\
\hline 1. & Arkadi yoga & Bn & Granthi & Kshira & 6 \\
\hline 2. & Dantimooladi taila & Lp & Granthi & Kshira & 20 \\
\hline 3. & Krishnadi lepa & Lp & Granthi & Kshira & 39 \\
\hline 4. & Dantyadi lepa & Lp & Granthi & Kshira & $3,11,12,13,16$ \\
\hline 5. & Granthi bhedana lepa & Lp & Granthi & Kshira & 6 \\
\hline 6. & Mahamarichadi taila & Tl & Granthi & Kshira & 35 \\
\hline
\end{tabular}

Table 11: Snuhi as an ingredient of various formulations indicated in Galganda (Goitre)

\begin{tabular}{|l|l|l|l|l|l|}
\hline Sr.No. & Formulation & Dosage Forms & Rogadhikara & Part used & References \\
\hline 1. & Dantyadi lepa & Lp & Galganda & Kshira & 10 \\
\hline 2. & Khadiradi lepa & Lp & Galganda & Kshira & 12 \\
\hline 3. & Dantimooladi lepa & Lp & Galganda & Kshira & 18 \\
\hline 4. & Shyamadi lepa & Lp & Galganda & Kshira & $4,12,18$ \\
\hline 5. & Snuhigandiradi sweda & Sd & Galganda & Kshira & 10 \\
\hline 6. & Shyamadi lepa & Lp & Medaja galganda & Kshira & $4,12,18$ \\
\hline
\end{tabular}


International Journal of Ayurvedic Medicine, 2017, 8(2), 45-57

Table 12: Snuhi as an ingredient of various formulations indicated in Pama (Scabies)

\begin{tabular}{|l|l|l|l|l|l|}
\hline Sr. No. & Formulation & Dosage Forms & Rogadhikara & Part used & References \\
\hline 1. & Brihata marichyadi taila & Ab & Pama & Kshira & 16 \\
\hline 2. & Adrakadi taila & Lp & Pama & Kshira & 12 \\
\hline 3. & Nisadi lepa & Lp & Pama & Patra & 13,20 \\
\hline 4. & Haridradi udvartana & Uv & Pama & Kshira & 20 \\
\hline 5. & Nishadi udvartana & Uv & Pama & Kshira & 11,12 \\
\hline 6. & Gunjadi udvartana & Uv & Pama & Kshira & 32 \\
\hline
\end{tabular}

Table 13: Snuhi as an ingredient of various formulations indicated in various other conditions

\begin{tabular}{|c|c|c|c|c|c|}
\hline Sr. No. & Formulation & Dosage Forms & Rogadhikara & Part used & References \\
\hline 1. & Korakadi yoga & $\mathrm{Tl}$ & Agnidaha & Kshira & 32 \\
\hline 2. & Karanja taila & $\mathrm{Tl}$ & Antra vidradhi & Patra & 25 \\
\hline 3. & Gunjadi lepa & Lp & Apachi & Kshira & 32 \\
\hline 4. & Maha ajmodadi taila & $\mathrm{Ns}, \mathrm{Ab}$ & Apachi & Kshira & 20 \\
\hline 5. & Gunjadi yoga & $\mathrm{Tl}$ & Apachi & Kshira & 32 \\
\hline 6. & Apara patana dravya & $\mathrm{Sn}$ & Apara patana & Kshira & 4 \\
\hline 7. & Yastimadhu adi yoga & Anjana,Ns & Apasmara & Kshira & 17 \\
\hline 8. & Dantyadi lepa & $\overline{L p}$ & Arbuda & Kshira & 39 \\
\hline 9. & Snuhi gandirikadi sweda & $\overline{\mathrm{Sd}}$ & Arbuda & Kanda & $\begin{array}{l}11,12,16,17,18, \\
39\end{array}$ \\
\hline 10. & Tiladi lepa & Lp & Arushika & Kshira & 5 \\
\hline 11. & Snuhikshiradi lepa & Lp & Arushika & Kshira & 13 \\
\hline 12 . & Snuhiadi taila & $\mathrm{Tl}$ & Arushika & Kshira & 13 \\
\hline 13. & Vajri lepa & $\overline{L p}$ & Bhagna & Kshira & 32 \\
\hline 14. & Vajri kshira & Lp & Galasundi & Kshira & 10,12 \\
\hline 15 . & Snuka-kshiradi lepa & Lp & Galasundi & Kshira & 12 \\
\hline 16. & Dantyadi lepa & Lp & Gandamala & Kshira & 22 \\
\hline 17. & Bhallatakadi lepa & $\overline{L p}$ & Gandamala & Moola & $16,17,20,22$ \\
\hline 18. & Ajmodaadi taila & $\mathrm{Tl}$ & Gandamala & Kshira & $12,16,20$ \\
\hline 19. & Snuka kshira yoga & Ks & Garbha salya & Kshira & 20 \\
\hline 20 . & Yoni dhupana yoga & $\mathrm{Dp}$ & Garbhasanga & Kshira & 6 \\
\hline 21. & Suhikshira yoga & Ks & Garbhini & Kshira & 25 \\
\hline 22. & Madanadi varti & $\mathrm{Vr}$ & Gulma & Kshira & 13 \\
\hline 23. & Snuhi dugdhadi tail & $\overline{\mathrm{Ab}}$ & Indralupta & Kshira & 13 \\
\hline 24. & Gunjadi yoga & $\mathrm{Tl}$ & Indralupta & Kshira & 43 \\
\hline 25. & Dhatrayadi lepa & Lp & Kacchu & Kshira & 20 \\
\hline 26. & Kachu rakshasa taila & $\overline{\mathrm{Ab}}$ & Kachu & Kshira & $15,18,20$ \\
\hline 27. & Snuhi arkadi lepa & $\overline{L p}$ & Kandu & Kshira & 20 \\
\hline 28. & Snukadi yoga & $\overline{L p}$ & Kantaka vidha & Kshira & 23 \\
\hline 29. & Snuhi kshira yoga & $\operatorname{Pr}$ & Karna roga & Kshira & 28 \\
\hline 30. & Dantyadi lepa & Lp & Karna sannipata & Kshira & 17 \\
\hline 31. & Vishagarbha taila & $\mathrm{Tl}$ & Karna soola & Kshira & 13,20 \\
\hline 32. & Shyamadi lepa & Lp & Karnika patana & Kshira & 5 \\
\hline 33. & Vajrikshiradi taila & $\mathrm{Tl}$ & Kesa ranjana & Kshira & 36 \\
\hline
\end{tabular}


Shashi Gupta et.al., Canvassing the External Applications of Snuhi: A Classical Memoir

\begin{tabular}{|c|c|c|c|c|c|}
\hline Sr. No. & Formulation & Dosage Forms & Rogadhikara & Part used & References \\
\hline 34. & Snuhidugdhadi lepa & Lp & Khalitya & Kshira & 17 \\
\hline 35. & Snuhiadi taila & $\mathrm{Tl}$ & Khalitya & Kshira & $\begin{array}{l}10,11,12,13,16,18,20, \\
32\end{array}$ \\
\hline 36. & Arkadi taila & $\mathrm{Tl}$ & Khalli & Kshira & $17,20,32$ \\
\hline 37. & Vishadi lepa & Lp & Kitibha & Kshira & 13 \\
\hline 38. & Chakramardadi lepa & Lp & Kitibha & Kshira & 12,18 \\
\hline 39. & Kitibhahara yoga & Lp & Kitibha & Kshira & 44 \\
\hline 40. & Beejapurakadi churna & $\mathrm{Cr}$ & Krimidanta & Kshira & 13 \\
\hline 41. & $\begin{array}{l}\text { Pratiksharniya Kshaar } \\
\text { yoga }\end{array}$ & Ks & Kshara karma & Kshira & 4 \\
\hline 42. & Ksharapaka & Ks & Kshara karma & Kshira & 17 \\
\hline 43. & Snukkshiradi lepa & Lp & Kunakha & Kshira & 32 \\
\hline 44. & Sumukhadi taila & $\overline{\mathrm{Ab}}$ & Masaka & Kanda & 20 \\
\hline 45. & Kshara ghrita & $\overline{\mathrm{Gr}}$ & Masaka & Kshira & 18,20 \\
\hline 46. & Arkadi lepa & Lp & Masaka & Kanda & 20 \\
\hline 47. & Snuhi bhavana & $\mathrm{Sp}$ & Masurika & Plant & 10,11 \\
\hline 48. & Snuhikshira yoga & $\overline{\mathrm{Sn}}$ & Mrita garbha & Kshira & 18 \\
\hline 49. & Snuhi kshira & Ks & Mudha garbha & Kshira & 28 \\
\hline 50. & Saktukadi lepa & Lp & Pada daha & Kshira & 28 \\
\hline 51. & Snuhikshiradi lepa & Lp & Pada sphutana & $\begin{array}{l}\text { Kshira,Ka } \\
\text { nda }\end{array}$ & 12 \\
\hline 52. & Snukkshiradi lepa & Lp & Padatala & Kshira & 25 \\
\hline 53. & Snuhi taila & $\mathrm{Tl}$ & Padatala & Kshira & 20 \\
\hline 54. & Vajridugdha yoga & $\mathrm{Sn}$ & Prasuta & Kshira & 22 \\
\hline 55. & Yoni vishodhana yoga & Dr & Raktagulma & Kshira & 3,5 \\
\hline 56. & Ksharadi palala & $\overline{\mathrm{Dr}}$ & Raktagulma & Kshira & 6 \\
\hline 57. & Ikshwaku adi yoga & $\overline{\mathrm{Dr}}$ & Raktagulma & Kshira & 12 \\
\hline 58. & Sudhakshira varti & $\mathrm{Vr}$ & Raktagulma & Kshira & 18 \\
\hline 59. & Sankhadi lepa & Lp & Romshatana & Kshira & $4,12,13$ \\
\hline 60. & Mahavisha garbha taila & $\overline{\mathrm{Ab}}$ & Sarva roga & Kshira & 39 \\
\hline 61. & Jivanarayana taila & $\mathrm{Ab}$ & Sarva roga & Kshira & 44 \\
\hline 62. & Rudra taila & $\mathrm{Tl}$ & Shiroroga & Moola & 20 \\
\hline 63. & Snuhi sweda & $\overline{\mathrm{Sd}}$ & Shlipada & Kanda & 20 \\
\hline 64. & Kshudradi lepa & $\mathrm{Lp}$ & Sirovedana & Kshira & 18 \\
\hline 65. & Snugadi yoga & $\mathrm{Bn}$ & Snayuka & Kshira & 23 \\
\hline 66. & Sudhayoga & Lp & Snayuka & Kshira & 17 \\
\hline 67. & Tapta raja taila & $\mathrm{Ab}$ & Sotha & Kshira & 18 \\
\hline 68. & Shaileyadi taila & $\mathrm{Ab}, \mathrm{Lp}$, Snana & Sotha & Kshira & 6 \\
\hline 69. & Gomutradi yoga & Lp & Sotha & Kshira & 44 \\
\hline 70. & Vachadi yoga & Lp & Stri roga & Kshira & 44 \\
\hline 71. & Ikshwaku yoga & $\mathrm{Vr}$ & Stri roga & Kshira & 20 \\
\hline 72. & Kinvadya varti & $\mathrm{Vr}$ & Stri roga & Kshira & 20 \\
\hline 73. & Ikshwaku adi varti & $\mathrm{Vr}$ & Stri roga & Kshira & 11,16 \\
\hline 74. & Sudhakshiradi lepa & $\mathrm{Lp}$ & Udara & Kshira & 17 \\
\hline 75 . & Mutra varti & $\mathrm{Vr}$ & Udara & Kshira & 4 \\
\hline 76. & Anaha varti & $\mathrm{Vr}$ & Udara & Kshira & 4 \\
\hline
\end{tabular}


International Journal of Ayurvedic Medicine, 2017, 8(2), 45-57

\begin{tabular}{|c|c|c|c|c|c|}
\hline Sr. No. & Formulation & Dosage Forms & Rogadhikara & Part used & References \\
\hline 77. & Naracha rasa & $\overline{L p}$ & Udavarta & Kshira & 50 \\
\hline 78. & Pinyakadi varti & $\mathrm{Vr}$ & Udavarta & Kshira & 3 \\
\hline 79. & Hingwadi taila & Ns,Ab,Dp & Unmada & Kshira & 5 \\
\hline 80. & Vishatindukadi lepa & $\overline{L p}$ & Upadansa & Kshira & 18 \\
\hline 81. & Vishyandana taila & $\mathrm{Tl}$ & Upadansa & Kshira & 10 \\
\hline 82. & Snuhiarkadugdhadi yoga & $\mathrm{Vr}$ & Upadansa & Kshira & 10 \\
\hline 83. & Rudra taila & $\overline{\mathrm{Ab}}$ & $\begin{array}{l}\text { Urdhwajatru } \\
\text { gata kapha roga }\end{array}$ & Moola & 18 \\
\hline 84. & Dasamooladi taila & $\mathrm{Tl}$ & Urustambha & Kshira & 8,12 \\
\hline 85. & Kusthakalanala taila & $\mathrm{Ab}$ & Vatarakta & Kshira & 40 \\
\hline 86. & Saptamrita lepa & $\overline{L p}$ & Vatarakta & Kshira & 20 \\
\hline 87. & Brihata marichyadi taila & $\mathrm{Tl}$ & Vatarakta & Kshira & 16,17 \\
\hline 88. & Mahavrikshadi kalka & $\mathrm{K} 1$ & Vicharchika & Kanda & 5 \\
\hline 89. & Snuga gandadi lepa & Lp & Vicharchika & Kanda & $6,11,13,18$ \\
\hline 90. & Sarsapkalka lepa & Lp & Vicharchika & Kanda & 12 \\
\hline 91. & Brihatmarichadi taila & $\mathrm{Tl}$ & Vicharchika & Kshira & 40 \\
\hline 92. & Dantyadi lepa & Lp & Visarpa & Kshira & 5 \\
\hline 93. & Karanjadi taila & $\mathrm{Tl}$ & Visarpa & Kshira & $13,16,17,18,20$ \\
\hline 94. & Sudhadi lepa & Lp & Visphota & Moola & 12 \\
\hline 95. & Arkarasadi taila & $\mathrm{Tl}$ & Visuchika & Kshira & 13 \\
\hline
\end{tabular}

Table 14: Snuhi as an ingredient of various formulations indicated in Panchakarma

\begin{tabular}{|l|l|l|l|l|l|}
\hline Sr. No. & Formulation & Dosage Forms & Rogadhikara & Part used & References \\
\hline 1. & Brihata kasisadi taila & $\mathrm{Ab}$ & Arsha & Kshira & $15,20,51$ \\
\hline 2. & Kasisadi taila & $\mathrm{Ab}$ & Arsha & Kshira & $5,12,18,20,35,39$ \\
\hline 3. & Jyotismati adi taila & $\mathrm{Ab}$ & Bhagandara & Kshira & 6 \\
\hline 4. & Chitrakadi taila & $\mathrm{Ab}$ & Bhagandara & Kshira & 12,20 \\
\hline 5. & Aswaghnamooladi taila & $\mathrm{Ab}$ & Dadru & Kshira & 9 \\
\hline 6. & Snuhi dugdhadi taila & $\mathrm{Ab}$ & Indralupta & Kshira & 15 \\
\hline 7. & Kachu rakshasa taila & $\mathrm{Ab}$ & Kachu & Kshira & $15,18,20$ \\
\hline 8. & Mahavajraka taila & $\mathrm{Ab}$ & Kustha & Kshira & $4,5,6$ \\
\hline 9. & Kustha kalanala taila & $\mathrm{Ab}$ & Kustha & Kshira & 20 \\
\hline 10. & Kusthadi taila & $\mathrm{Ab}$ & Kustha & Kshira & $6,17,20$ \\
\hline 11. & Marichyadi taila & $\mathrm{Ab}$ & Kustha & Kshira & $11,13,20$ \\
\hline 12. & Kusthahara yoga & $\mathrm{Ab}$ & Kustha & Kshira & 44 \\
\hline 13. & Vajri taila & $\mathrm{Ab}$ & Kustha & Kshira & 20,32 \\
\hline 14. & Bhanu taila & $\mathrm{Ab}$ & Kustha & Kshira & 20,39 \\
\hline 15. & Vajraka taila & $\mathrm{Ab}$ & Kustha & Kshira & $4,5,10,12,14,16,17$ \\
\hline 16. & Lakwahara taila & $\mathrm{Ab}$ & Lakwa & Kshira & 44 \\
\hline 17. & Sumukhadi taila & $\mathrm{Ab}$ & Masaka & Kanda & 20 \\
\hline 18. & Brihata marichyadi taila & $\mathrm{Ab}$ & Pama & Kshira & 16 \\
\hline 19. & Mahavisha garbha taila & $\mathrm{Ab}$ & Sarva roga & Kshira & 39 \\
\hline 20. & Jivanarayana taila & $\mathrm{Ab}$ & Sarva roga & Kshira & 44 \\
\hline 21. & Tapta raja taila & $\mathrm{Ab}$ & Sotha & Kshira & 18 \\
\hline 22. & Rudra taila & $\mathrm{Ab}$ & $\begin{array}{l}\text { Urdhwajatru ga- } \\
\text { ta kapha roga }\end{array}$ & Moola & 18 \\
\hline 23. & Kusthakalanala taila & $\mathrm{Ab}$ & Vatarakta & Kshira & 40 \\
\hline 24. & Vishatinduka taila & $\mathrm{Ab}$ & Vatavyadhi & Kshira & 18 \\
\hline 25. & Visha tindukataila & $\mathrm{Ab}$ & & 20 \\
\hline
\end{tabular}


Shashi Gupta et.al., Canvassing the External Applications of Snuhi: A Classical Memoir

\begin{tabular}{|c|c|c|c|c|c|}
\hline Sr. No. & Formulation & Dosage Forms & Rogadhikara & Part used & References \\
\hline 26. & Atharanga taila & $\mathrm{Ab}$ & Vatavyadhi & Patra & 44 \\
\hline 27. & Shaileyadi taila & $\mathrm{Ab}$ & Sotha & Kshira & 6 \\
\hline 28. & Maha Narayana taila & $\mathrm{Ab}$ & Vatavyadhi & Moola & 15 \\
\hline 29. & Mahamarichadi taila & $\mathrm{Ab}$ & Kustha & Kshira & $10,11,12,18,39$ \\
\hline 30. & Mahamarichadi taila & $\mathrm{Ab}$ & Kustha & Kshira & 15 \\
\hline 31. & Shwitragajasigha taila & $\mathrm{Ab}$ & Kustha & Kshira & 35 \\
\hline 32. & Gaja asthiadi taila & $\mathrm{Ab}$ & Arsha & Kanda & 5 \\
\hline 33. & Maha ajmodadi taila & $\mathrm{Ab}$ & Apachi & Kshira & 20 \\
\hline 34. & Hingwadi taila & $\mathrm{Ab}$ & Unmada & Kshira & 5 \\
\hline 35. & Snuhi gandirikadi sweda & $\mathrm{Sd}$ & Arbuda & Kanda & $\begin{array}{l}11,12,16,17,18, \\
39\end{array}$ \\
\hline 36. & Snuhigandiradi sweda & $\mathrm{Sd}$ & Galganda & Kshira & 10 \\
\hline 37. & Vishadi yoga & $\mathrm{Sd}$ & Kustha & Khsira & 10,12 \\
\hline 38. & Snuhi sweda & $\mathrm{Sd}$ & Shlipada & Kanda & 20 \\
\hline 39. & Erandadi yoga & $\mathrm{Sd}$ & Vatavyadhi & Kshira & 16,17 \\
\hline 40. & Gunjadi yoga & $\mathrm{Uv}$ & Dadru & Kshira & 17 \\
\hline 41. & Prapunnadi udvartana & Uv & Kustha & Kshira & 11 \\
\hline 42. & Gunjadi udvartana & Uv & Kustha & Kshira & 16 \\
\hline 43. & Snuhyadi udvartana & Uv & Kustha & Kshira & 25 \\
\hline 44. & Vajra shekhara rasa & $\mathrm{Uv}$ & Kustha & Kshira & 46 \\
\hline 45. & Nishadi yoga & $\mathrm{Uv}$ & Kustha & Patra & 10 \\
\hline 46. & Haridradi udvartana & $\mathrm{Uv}$ & Pama & Kshira & 20 \\
\hline 47. & Nishadi udvartana & $\mathrm{Uv}$ & Pama & Kshira & 11,12 \\
\hline 48. & Gunjadi udvartana & $\mathrm{Uv}$ & Pama & Kshira & 32 \\
\hline 49. & Maha Narayana taila & $\mathrm{Bs}$ & Vatavyadhi & Moola & 15 \\
\hline 50. & Maha Narayana taila & $\mathrm{Ns}$ & Vatavyadhi & Moola & 15 \\
\hline 51. & Mahamarichadi taila & $\mathrm{Ns}$ & Kustha & Kshira & $10,11,12,18,39$ \\
\hline 52. & Mahamarichadi taila & Ns & Kustha & Kshira & 15 \\
\hline 53. & Yastimadhu adi yoga & Ns & Apasmara & Kshira & 17 \\
\hline 54. & Sirisapuspadi yoga & Ns & Manduka visha & Kshira & 28 \\
\hline 55. & Maha ajmodadi taila & Ns & Apachi & Kshira & 20 \\
\hline 56. & Hingwadi taila & $\mathrm{Ns}$ & Unmada & Kshira & 5 \\
\hline
\end{tabular}

\section{Discussion:}

One of the main causes for skin ailments is infecton. The methanol extract of plant Euphorbia neriifolia possess significant antimicrobial action (57). Snuhi latex is widely used in prepration of Ksharasutra for the treatment of Arsha, snuhi latex acts as a binding agent, having almost all the apamarga kshara properties intact, which in fact liberates many fold of medicament having surgical actions like incision, excision debridation, scrapping and medical action like hemostatic, antiseptic, healing, etc., which act simultaneously to cure the arsha (58). Latex of snuhi is widely used in management of wound. Wound healing activity of aqueous extract of E. neriifolia has been reported (59). Many ethno-medicinal practices, prevelance today, also suggests that the use of Snuhi as antidote in scorpion bite, snake bite (60). Snuhi Kshira is indicated in sotha and anti inflammatory and analgesic activity of $70 \% \mathrm{v} / \mathrm{v}$ hydroalcoholic extract of dried leaves of $E$. neriifolia, by oral administration, at dose of $400 \mathrm{mg} / \mathrm{kg} /$ day of body weight to healthy albino rats has been reported (61). In relation with treatment of Unmada, psychopharmacological profile of hydroalcoholic extracts of $E$. neriifolia leaves in mice and rats, the leaf extract significantly reduces apomorphine induced stereotypyin mice at all doses (100, 200, $400 \mathrm{mg} / \mathrm{kg}$ body weight) (62). Apart from this, Snuhi has been advocated in various procedures of Panchakarma like Abhyanga, Swedana, Niruha basti, Nasya, etc. mainly in treatise.(63).

\section{Conclusion:}

Snuhi finds a wide range of therapeutic importance in 253 external applications in almost 62 disease conditions. Moreover, its usage in panchakarma inspite of being included in semi-poisonous plant 
category, again draw attention to its safe local administration. More thorough clinical data can suffice the validation of these external uses of Snuhi.

\section{References:}

1. Mishra Sadananda. Rasatrangani, Shastri Kashinath, Editor. Motilal Banarasidas, Varanasi; 2000. p 676.

2. Charaka Samhita. Pt.Rajeswara Datta Shastri, Editor. Kalpasthana Chaukhambha Bharati Academy Reprinted 2011. P 933.

3. Charaka Samhita. Pt.Rajeswara Datta Shastri, Editor. Kalpasthana Chaukhambha Bharati Academy Reprinted 2011.

4. Sushruta. Sushrutasamhita. Kaviraj Ambika Dutta Shashtri. Vol I \& II, Chaukhambha Sanskrit Sansthan,Varanasi, Reprint 2012.

5. Vaghbhatt. Astanga Sangraha. Atrideva Gupta, Editor. Vol. I \& II, Chaukhamba Krishnadas Academy, Varanasi; Printed 2005.

6. Vaghbhatt. Astanga Hridaya. Atrideva Gupta, Editor. Chaukhambha Prakashan, Varanasi, Rerprint 2012

7. Kashyapa. Kashyapa Samhita. P.V. Tewari, Editor, Chaukhamba Vishwabharti, Varanasi; 2008.

8. Maharshi Bhela, Bhela Samhita. Prof. Priya vrat Sharma, Editor, Chaukhamba Visvaabharati, Varanasi, 2006.

9. Harihar Prasad Tripathi, Editor, Harita Samhita. Chaukhamba Krishnadas Academy, Varanasi; 2009.

10. Tiwari P, Editor. Vrindhamadhav. Chaukhamba Vishwabharti, Varanasi; 2007.

11. Indradeva Tripathi, Editor. Chakradatta. Chaukhamba Sanskrit Bhawan, Varanasi; 2014.

12. Shodhala. Gadanigraha. Indradeva Tripathi, Editor.Vol.I, II \& III.Chaukhamba Sanskrit Sanstana, Varanasi; Edition 2012.

13. Vangasen. Vangasen. Pt. Harihar Prashada Tripathi, Editor, Chaukhambha KrishnaDas Academy, Varanasi; Edition 2009.

14. Sharandhara. Sharandhara Samhita. Dr. Smt. Shailaja Srivastava, Editor. Chaukhamba Orientalia, Varanasi; Edition 2013.

15. Bhavamishra. Bhavaprakasha Samhita. Bhramhashanankar Mishra Shashrina, Editor. Chaukhamba Sanskrit Bhawan, Varanasi; 2013.

16. Shashtri Laxmipati. Yogratnakara. Bhrahmasankar Shashtri, Editor. Chaukhamba Prakashana, Varanasi; Edition 2013.

17. Bhrihatnighanturatnakar.

Shrikrishnalal Madhukar, krishnadas prakashana; 2011.

18. Govindadasa. Bhaishajya Ratnavali. Ambikadatta Shashtri, Editor. Chaukhamba Prakashan, Varanasi; Reprinted 2011.

19. Nishteswar K. \& Vidyanath. R. Sahasrayogam, Chowkhamba Krishnadas Academy, Varanasi; 2006

20. Shah Naginadasa Chhaganlal, Bharat Bhaishajya Ratnakar. Vol I to Vol V. First edition B. Jain Publishers, New Delhi; 1999.

21. Tistacharya. Chikitsa kalika. Dr. Shri Vishal
Tripathi, Editor. Chaukhamba Orientalia, Varanasi; $1^{\text {st }}$ Edition 2000.

22. Lolimbaraja. Chamatkara Chintamani. Nirmal Saxena, Editor. Krishnadas Academy, Varanasi; 2001.

23. Bhatta Krishnarama, Siddhabheshaja Manimala. Chaukhamba Krishndas Academy, Varanasi; 2008.

24. Lolimbaraja. Vaidyajivan. Krishnaji Vishnu Joshi Vaidya, Prakashaka yageswara Gopal Dixit, Editor. Pune; 1930

25. Kalidasa. Vaidya manorama, Dr. S. Sharma, Editor. Chaukhambha Orientallia, Delhi; 2012.

26. Nagarjuna. Rasendramangala. Sharma HS, Editor. Chaukhamba Oriental, Varanasi; 2003.

27. Bhagvatpada Govinda. Rasa Hridaya Tantra, Rameshwar Daydlu Vajpayee, Editor. Krishnadas Academy, Varanasi; 1998.

28. Bhojraja. Rajamartanada. Prof. Siddhi Nandana Mishrra, Editor, Chaukhamba Orientalia, Varanasi; 2009.

29. Tripathi Indradeva, Editor. Rasarnava Nama Rasatantra. Chaukhamba Sanskrit series, Varanasi; 2012.

30. Somadeva. Rasendrachudamani. Bajpai RD, Editor. Chaukhamba Krishnadas Academy, Varanasi; 2004.

31. Yashodhara. Rasa Prakasha Sudhakara. Mishra Siddhinanda, Editor. Chaukhamba oriental Varanasi; 2013.

32. Vaghabhata. Rasaratnasammuchaya. Kapidev Giri, Editor. Chaukhambha Sanskrit Sansthan, Varanasi; 2012.

33. Bindu. Rasapaddhati. Dr. Siddhinandan Mishra, Editor. Chaukhambha Orientalia, Varanasi; 1987.

34. Bhairavakta Anandakanda. Mishra Siddhinanda, Editor. Chaukhamba orientalia, Varanasi; 2008.

35. Anantadev Suri. Rasachintamani. Mishra Siddhinanda, Editor. Chaukhamba Orientalia, Varanasi; 1990.

36. Shalinath. Rasamanjiri. Prof. Siddhi Nandan Mishra, Editor. Chaukhamba Orientalia, Varanasi; 1995.

37. Dhundhukanatha. Rasendrachintamani. Mishra Siddhinanda, Editor. Chaukhhamba oriental, Varanasi; 2011.

38. Rasendrasara Sangraha. Mishra Siddhinandan, Editor. Chaukhamba Oriental, Varanasi; 2010.

39. Mishra Chudamani. Rasa Kamadhenu. Mishra Gulrajasharma, Editor. Chaukhamba Oriental, Varanasi; 1999.

40. Trimalla Bhatta. Yogatarangini. Zha Chandrabhshana, Editor. Chaukhamba Vidyabhavan, Varanasi; 2003.

41. Lankapati Ravan. Arkaprakasha. Tripathi Indradev, Editor. Chaukhamba Krishnadas Academy, Varanasi; 2011.

42. Madhava. Ayurveda Prakasha, Mishra Gulrajasharma. Editor. Chaukhamba Bharati Academy, Varanasi; 2007.

43. Bhrihat Rasarajasundara. Dattarama Chaube, Editor. Chaukhamba oriental, Varanasi; 2000.

44. Dasa Bhagwana. Rasaraj Mahodadhi. Khemaraja Shri Krishna Dasa Academy. Mumbai; 2012. 
45. Rasa Jala Nidhi, Bhudeb Mookerjee.Vol. 1,2,3,4 \& 5. Chaukhambha Publishers.Varanasi; 1999.

46. Sharma Hariprapanna, Rasayogasagara. Vol.I \& II, Chaukhamba Krishnadas Academy, Varanasi; 2010.

47. Sharma Trayambaknath, Rasamitra. Chaukhamba Sanskrita series, Varanasi; 2001.

48. Badrinarayana Sharma, Rasopanishata. Krishna Gopal Mudranalaya; 1956.

49. Mishra Sadananda. Rasatrangani, Shastri Kashinath, Editor. Motilal Banarasidas, Varanasi; 2000.

50. Dwivedi Vishwanath, Rasendra Sambhava. First edition, Krishnadas Academy, Varanasi; 1997

51. Mishra Siddhinandana, Editor. Abhinava Navajeevaniyama. Chaukhamba Orentalia, Varanasi; 2000.

52. Sharma Dnyanachandra. Rasa Kaumudi. Sharma Pavani Prasad, Editor. Sampadaka bhishajaratna Shri Bhramashankar Mitra, Chaukhamba Bharati Academy, Varanasi, 2003.

53. Kayasta Chamunda. Rasa Sanketa Kalika. Shastri Satyanarayan Pammi, Chaukhamba Krishnadas Academy; 2005.

54. Vajpayee Rameshwar Dayal, Rasa Dipika. First edition. Chaukhamba Krishnadas Academy, Varanasi; 2003.

55. A dictionary English and Sanskrit: M. Monier Williams, Motilal Banarsidas Publishers Private Limited, Delhi - Reprint - 1999 Office, Varanasi, Shabdkalpadruma, 1961.

56. Anonymous. The Ayurvedic Formulary of India ebook Part 1. New Delhi: Govt. of India: Ministry of Health and Family Welfare, Department of Indian
Systems of Medicine and Homoepathy; 2003.

57. Datta Samaresh, Nayak Siva and Dinda Subas, Exploration of Antimicrobial Potential of Methanol Extract of Stems of Euphorbia Neriifolia. IRJP 2013, 4 (1)

58. Meva Lal Gupta, S.K. Gupta and Chaturbhuja Bhuyan, Comparative clinical evaluation of Kshara Sutra ligation and hemorrhoidectomy in Arsha (hemorrhoids) Ayu. 2011 Apr-Jun; 32(2): 225-229.

59. Shaikh Arshad Ahmed, Sayyed Nazim, Shaikh Siraj, Patel M. Siddik and Chavda Ab. Wahid, Euphorbia neriifolia Linn: A phytophormacological review; International Research Journal of Pharmacy IRJP, 2011; 2(5): 41-48

60. Rev. FR. Jean Ferdinand Caius, The Medicinal and poisonous plants Of India, Scientfic publishers, Jodhpur, 2003.

61. Shaikh Arshad Ahmed, Sayyed Nazim, Shaikh Siraj, Patel M. Siddik and Chavda Ab. Wahid, Euphorbia neriifolia Linn: A phytophormacological review; International Research Journal of Pharmacy IRJP, 2011; 2(5): 41-48

62. Bigoniya P, Rana AC; Psychopharmacological profile of hydroalcoholic extract of Euphorbia neriifolia leaves in mice and rats; Indian J Exp Biol 2005; 43(10): 859-862.63.

63. Bhavamishra. Bhavaprakasha Samhita. Bhramhashanankar Mishra Shashrina. Editor. Chaukhamba Sanskrit Bhawan, Varanasi; 2013.

64. Shashi Gupta and Rabinarayan Acharya, An appraisal on ethno-medicinal claims of Euphorbia caducifolia Haines. - a source drug of ayurvedic medicinal plant 'Rakta-snuhi', Global J Res. Med. Plants \& Indigen. Med., 2017, pp. 54-63. 